\title{
FULL-COMPLETENESS IN WEIGHTED SPACES
}

\author{
W. H. SUMMERS
}

1. Introduction. That the class of fully complete ( $B$-complete) locally convex spaces has an essential role has been well-established (see, e.g., Collins [2], Husain [12], and Pták [16]). However, it is still the case that very little is known concerning full-completeness in function spaces except when implied by formally stronger properties (e.g., Fréchet), and our aim in this article is to shed some light in this direction. Our approach is to use the unifying notion of the weighted spaces $C V_{0}(X)$ discussed in $[\mathbf{1 9} ; \mathbf{2 0}]$, since such spaces provide a general setting for the study of virtually all continuous function spaces encountered in analysis.

The results to be presented here divide naturally into three sections with $\S 2$ being consigned the preliminary definitions and theorems. In $\S 3$ we obtain a necessary condition for full-completeness in a class of function spaces, and consider certain consequences of this result. This necessity theorem includes a result implicit in a paper by Pták [15], as well as the important case of $\left(C_{b}(X), \beta\right)$, the space of all bounded continuous functions on a locally compact Hausdorff space endowed with the strict topology $\beta$ (see Buck [1]). Several conditions are given in $\S 4$ which are necessary and sufficient for the space $\left(C_{b}(X), \beta\right)$ to be $B_{r}$-complete, while $\S 5$ is devoted to the consideration of full-completeness in products and tensor products of function spaces. In this section we give a detailed account of an example announced in [18] which shows that the product of two fully complete spaces (actually two spaces with the Krein-Smulian property [4, p. 364]) need not be fully complete, and consequently resolve a question of some significance; see, e.g., Kelley [13] or Husain [12].

Acknowledgement. I wish to express my appreciation to Professor Collins for his advice and encouragement.

2. Preliminaries. The underlying topological spaces $(X$ and $Y)$ in the following will be at least completely regular $T_{1}$-spaces, while all functions will be complex-valued unless explicitly stated otherwise. We shall let $C(X)$ denote the space of all continuous functions on $X, B(X)$ denote the space of all bounded functions on $X$, and $B_{0}(X)$ denote the space of all functions on $X$ which vanish at infinity. Further, let $C_{b}(X)=C(X) \cap B(X)$ and

Received February 10, 1970. This research was partially supported by National Science Foundation grant GP-11762. Several of the results in this paper are from the author's Ph.D. dissertation written under the direction of Professor Heron S. Collins at Louisiana State University in 1968. 
$C_{0}(X)=C(X) \cap B_{0}(X)$, while $C_{c}(X)$ will denote the space of all continuous functions on $X$ which have compact support. If $f$ is a function on $X$, then $N(f)=\{x \in X: f(x) \neq 0\}$ and the closure of $N(f)$ is denoted spt $(f)$. Moreover, if $S \subseteq X$, then $R(f ; S)$ will denote the restriction of $f$ to $S$, and if $R(f ; S) \in B(S)$, then define $\|f\|_{S}=\sup \{|f(x)|: x \in S\}$. In the case $S=X$ we write $\|f\|$, and will refer to the topology induced by $\|\cdot\|$ on $B(X)$ as the uniform topology.

A Nachbin family $V$ on $X$ is a set of non-negative upper semicontinuous (u.s.c.) functions on $X$ satisfying the condition that if $u$, $\in \in V$ and $\lambda \geqq 0$, then there is a $w \in V$ such that $\lambda u, \lambda v \leqq w$ (pointwise). If $V$ is a Nachbin family on $X$, then the corresponding weighted space

$$
C V_{0}(X)=\left\{f \in C(X): f v \in B_{0}(X) \text { for every } v \in V\right\}
$$

endowed with the weighted topology $\omega$ (or $\omega_{V}$ ) generated by the seminorms $\left\{p_{v}: v \in V\right\}$, where $p_{v}(f)=\|f v\|$ for each $f \in C V_{0}(X)$. Now $C V_{0}(X)$ is a locally convex (topological vector) space with a base of closed and absolutely convex neighbourhoods of zero formed by the sets

$$
V_{v}=\left\{f \in C V_{0}(X):\|f v\| \leqq 1\right\}
$$

for each $v \in V$. If $U$ and $V$ are two Nachbin families on $X$ and if for each $u \in U$ there is a $v \in V$ such that $u \leqq v$, then we write $U \leqq V$. In case $U \leqq V$ and $V \leqq U$, we write $U \approx V$.

Unless otherwise specified, we will consider only Nachbin families $V$ on $X$ for which $C V_{0}(X)$ is Hausdorff (necessary and sufficient conditions for this, as well as examples of weighted spaces are given in [19]). In fact, we will normally assume a slightly stronger separation property on $C V_{0}(X)$ (as will become apparent in $\S 3$ ) but one which is generally attainable in practice.

We will conclude this section with some general results on the theory of fully complete spaces (our notation and terminology concerning this subject will basically be that of $[8]$ ). The following (surely known) corollary to Pták's open mapping theorem is stated and a proof sketched here since it will be used several times in the paper.

2.1. Theorem. Let $E$ and $F$ be locally convex spaces (here, and throughout this paper, a locally convex space is a locally convex Hausdorff topological vector space) and let $t: E \rightarrow F$ be a linear map with the graph $G_{t}$ of $t$ closed in $E \times F$. If $E$ is fully complete and if $t$ is nearly open, then $t$ is open onto $F$. If, in addition, $t$ is one-to-one, then it suffices for the conclusion that $E$ be $B_{r}$-complete.

Proof. Let $H=E / t^{-1}(0)$ and let $u: H \rightarrow F$ be the map induced by $t$. Since $H$ is fully complete [2, p. 276], Pták's open mapping theorem [16, p. 57] yields that $u$ is open onto $u(H)$ with the relative topology. If $y \in F$, then there is a net $\left\{y_{i}\right\}$ in $u(H)$ such that $y_{i} \rightarrow y$, and hence $\left\{u^{-1}\left(y_{i}\right)\right\}$ is a Cauchy net in $H$. Consequently, there is an $x \in H$ such that $u^{-1}\left(y_{i}\right) \rightarrow x$, and since $G_{u}$ is closed in $H \times F$, the result follows. 
We also note the following generalization of a result due to Collins [2, p. 275]. The proof follows as in [2] after an application of Theorem 2.1.

2.2. Theorem. Let $E$ and $F$ be locally convex spaces and let $t: E \rightarrow F$ be linear and nearly open. If $E$ is fully complete and if $t$ is weakly continuous, then $t$ is open onto $F$ and $F$ is fully complete.

2.3. Theorem. If $E$ is a Mackey space (i.e., a locally convex space with the Mackey topology $\left.\tau\left(E, E^{*}\right)\right)$, then $E$ is fully complete if and only if every continuous nearly open linear map into a Mackey space is open and onto.

Proof. If $E$ is fully complete, then the result follows immediately from Theorem 2.1. For the converse, let $L$ be a nearly closed linear subspace of the topological dual $E^{*}$ of $E$. Consider $F=E / L^{0}$ endowed with the topology of uniform convergence on

$$
\left\{U^{0} \cap L: U \in \mathscr{U}\right\},
$$

where $\mathscr{U}$ is a base of absolutely convex neighbourhoods of the origin in $E$, and note that $F^{*}=L$. Since the quotient topology on $F$ is finer than the topology defined above, the quotient map $q: E \rightarrow F$ is continuous. Therefore if $A$ is any absolutely convex and $\sigma(L, F)$-compact subset of $L$, then $A$ is $\sigma\left(E^{*}, E\right)$ compact, and this implies that there is a $U \in \mathscr{U}$ such that $A \subseteq U^{0}$ whence $A \subseteq U^{0} \cap L$. It now follows from the Mackey-Arens theorem that $F$ is a Mackey space, and hence $q$ is open onto $F$ since a routine calculation shows that $q$ is nearly open. Consequently, $F$ has the quotient topology implying that $L=F^{*}=L^{00}$, and this implies that $L$ is $\sigma\left(E^{*}, E\right)$-closed which completes the proof.

3. A condition necessary for full-completeness. If $V$ is a Nachbin family on $X$ and if $F$ is any closed subspace of $X$, then

$$
R_{F}(V)=\{R(v ; F): v \in V\}
$$

is easily seen to be a Nachbin family on $F$, and $C R_{F}(V)_{0}(F)$ is Hausdorff for every closed subspace $F$ of $X$ if and only if $V$ satisfies:

$(\alpha)$ for each $x \in X$ there is a $v \in V$ such that $v(x)>0$.

If $V$ satisfies $(\alpha)$, then $\omega_{V}$ is finer than the topology of pointwise convergence on $C V_{0}(X)$. The following example shows that the converse of the preceding statement is false, and also has the virtue of showing that [19, Theorem 3.3] cannot be substantially improved.

3.1. Example. If we let $X$ denote the rationals in $[0,1]$ with the topology induced by the usual topology on the reals and if $V=\left\{v \in C_{b}+(X): v(1)=0\right\}$, then $V$ is a Nachbin family on $X$ which fails to satisfy $(\alpha)$ and yet $\omega_{V}$ is clearly at least as fine as the topology of pointwise convergence on $C V_{0}(X)$.

We will assume throughout the remainder of this section that a Nachbin family on $X$ satisfies $(\alpha)$ unless otherwise stated. Also, in the following, we 
will denote the mapping $f \rightarrow R(f ; F)$ by $R(\cdot ; F)$ where $f$ is a function on $X$ and $F$ is a subset of $X$.

3.2. Lemma. Let $V$ be a Nachbin family on $X$ and let $F$ be a closed subspace of $X$. Then $R(\cdot ; F)$ is a continuous linear mapping of $C V_{0}(X)$ into $C R_{F}(V)_{0}(F)$.

Proof. This is an immediate consequence of the definitions of weighted spaces and the Nachbin family $R_{F}(V)$.

3.3. Lemma. Let $V$ be a Nachbin family on $X$ and let $F$ be a closed subspace of $X$. If either

(1) $v \in V$ implies that spt(v) is compact, or

(2) $X$ is locally compact and $V \leqq B^{+}(X)$, then $\left\{R(f ; F): f \in C V_{0}(X)\right\}$ is dense in $C R_{F}(V)_{0}(F)$.

Proof. $R(\cdot ; F)$ is a well-defined linear mapping of $C V_{0}(X)$ in to $C R_{F}(V)_{0}(F)$ by Lemma 3.2. Now assume that (1) holds; let $f \in C R_{F}(V)_{0}(F)$, let $v \in V$, and let $u=R(v ; F)$. If $u \neq 0$, then $\operatorname{spt}(u)$ is compact, and hence Stone's theorem $[\mathbf{1 0}$, p. 90$]$ yields a $g \in C_{b}(X)$ such that $R(g ; \operatorname{spt}(u))=R(f ; \operatorname{spt}(u))$. Therefore $|(R(g ; F)-f)(x)| u(x)=0$ for every $x \in F$, and since $g \in C V_{0}(X)$, our proof is complete in this case.

Assume that (2) holds, let $\mu \in C R_{F}(V)_{0}(F)^{*}$, and suppose that $\langle R(f ; F), \mu\rangle=0$ for every $f \in C V_{0}(X)$. We will show that $\mu=0$, and since $C_{c}(F)$ is dense in $C R_{F}(V)_{0}(F)[\mathbf{1 4}, \mathrm{p} .64]$, it will suffice to show that $\langle\phi, \mu\rangle=0$ for every $\phi \in C_{c}(F)$. To this end, take $\phi \in C_{c}(F)$ with $\phi \neq 0$ and let $\epsilon>0$. It follows from [20, Theorem 3.1] that $\mu$ can be assumed to be a bounded Radon measure on $F$, and hence there is a compact set $K$ in $F$ such that $|\mu|(F \backslash K)<\epsilon\left(\|\phi\|_{F}\right)^{-1}$. If $G=K \cup \operatorname{spt}(\phi)$, then Stone's theorem and Urysohn's lemma yield an $f \in C_{c}(X)$ such that $R(f ; G)=R(\phi ; G)$ and we may assume that $\|f\|=\|\phi\|_{F}$. Thus $f \in C V_{0}(X)$ and

$$
\begin{aligned}
|\langle\phi, \mu\rangle|=\left|\int_{F} \phi d \mu\right| & =\left|\int_{G} \phi d \mu+\int_{F \backslash G} R(f ; F) d \mu-\int_{F \backslash G} R(f ; F) d \mu\right| \\
= & \left|\int_{F} R(f ; F) d \mu-\int_{F \backslash G} R(f ; F) d \mu\right|=\left|\int_{F \backslash G} R(f ; F) d \mu\right| \leqq \epsilon,
\end{aligned}
$$

from which we have that $\langle\phi, \mu\rangle=0$, and the proof is complete.

3.4. Lemma. Let $V$ be a Nachbin family on $X$ and let $F$ be a closed subspace of $X$. If either

(1) $v \in V$ implies that spt(v) is compact, or

(2) $X$ is locally compact and $V \leqq B^{+}(X)$, then $R(\cdot ; F)$ is a continuous nearly open linear mapping of $C V_{0}(X)$ into $C R_{F}(V)_{0}(F)$.

Proof. By Lemma $3.2, R(\cdot ; F)$ is a continuous linear mapping of $C V_{0}(X)$ into $C R_{F}(V)_{0}(F)$, while $\left\{R(f ; F): f \in C V_{0}(X)\right\}$ is dense in $C R_{F}(V)_{0}(F)$ by Lemma 3.3. Let $v \in V$, let $u=R(v ; F)$, and let

$$
A=\left\{f \in C R_{F}(V)_{0}(F):\|f u\|_{F}<1\right\} .
$$


Since $A$ is an open neighbourhood of zero in $C R_{F}(V)_{0}(F)$,

$$
A \cap\left\{R(g ; F): g \in C V_{0}(X)\right\}
$$

is non-void; and we will show that if $R(f ; F) \in A$ where $f \in C V_{0}(X)$, then we may assume that $f \in V_{v}$. Let $K=\{x \in X:|f(x)| v(x) \geqq 1\}$ and note that $f \in V_{v}$ if $K$ is void. Thus it suffices to assume that $K$ is not void, and in this case $K$ is compact and disjoint from $F$. It now follows from [14, p. 69] that there is a $\phi \in C(X)$ with $0 \leqq \phi \leqq 1, \phi(K)=0$, and $\phi(F)=1$; if $g=f \phi$, then $g \in C V_{0}(X)$ and $R(g ; F)=R(f ; F)$. Moreover, $x \in K$ implies that $|g(x)| v(x)=0$, while

$$
|g(x)| v(x)=|f(x)| \phi(x) v(x) \leqq|f(x)| v(x)<1 \text { if } x \notin K .
$$

Consequently, $g \in V_{v}$ and the claim is established. Thus we have

$$
A \cap\left\{R(f ; F): f \in C V_{0}(X)\right\} \subseteq\left\{R(f ; F): f \in V_{r}\right\}
$$

from which it follows that $A \subseteq \operatorname{cl}\left(\left\{R(f ; F): f \in V_{v}\right\}\right)$; i.e., $R(\cdot ; F)$ is nearly open.

3.5. Theorem. Let $V$ be a Nachbin family on $X$. If $C r_{0}(X)$ is fully complete and if either

(1) $v \in V$ implies that $\operatorname{spt}(v)$ is compact, or

(2) $X$ is locally compact and $V \leqq C_{0}{ }^{+}(X)$,

then $X$ is normal.

Proof. It will suffice to show that if $F$ is a closed subspace of $X$, then $F$ is $C^{*}$-embedded in $X[\mathbf{1 0}$, p. 48]. We have from Lemma 3.4 that $R(\cdot ; F)$ is a continuous nearly open linear mapping of $C V_{0}(X)$ into $C R_{F}(V)_{0}(F)$, and so $R(\cdot ; F)$ is open onto $C R_{F}(V)_{0}(F)$ by Theorem 2.1. It is easily verified that $f \in C_{b}(F)$ implies that $f \in C R_{F}(V)_{0}(F)$, and hence there is a $g \in C V_{0}(X)$ for which $R(g ; F)=f$. The proof is now complete, since in this case we may assume that $g \in C_{b}(X)$.

Before considering any specific applications of the preceding necessity theorem, we note that as a consequence of Lemma 3.4 and Theorem 2.2 we have the following result.

3.6. Theorem. Let $V$ be a Nachbin family on $X$ and let $F$ be a closed subspace of $X$. If $C V_{0}(X)$ is fully complete and if either

(1) $v \in V$ implies that spt(v) is compact, or

(2) $X$ is locally compact and $V \leqq B^{+}(X)$, then $C R_{F}(V)_{0}(F)$ is fully complete.

3.7. Theorem. If $C(X)$ with the compact-open (co-op) topology is fully complete, then $X$ is normal and $(C(F)$, co-op) is fully complete for every closed subspace $F$ of $X$. 
Proof. $V=\left\{\lambda \chi_{K}: \lambda \geqq 0, K \subseteq X, K\right.$ compact $\}$, where $\chi_{S}$ denotes the characteristic function of a subset $S \subseteq X$, is a Nachbin family on $X$ (which satisfies $(\alpha))$ and $C V_{0}(X)=(C(X)$, co-op). Moreover, if $F$ is a closed subspace of $X$, then $R_{F}(V)=\left\{\lambda \chi_{K}: \lambda \geqq 0, K \subseteq F, K\right.$ compact $\}$. The result now follows from Theorems 3.5 and 3.6.

The preceding theorem is a result implicit in a paper by Pták [15, Theorem 6.7]. Collins [3, p. 835] (and somewhat later, Warner [23]) has shown that if $X$ is a hemi-compact $k$-space, then $(C(X)$, co-op) is fully complete. However, before this paper there was little else known about full-completeness in function spaces. Before proceeding, we should note that if $(C(X \times I)$, co-op $)$ is fully complete where $I=[0,1]$, then as a corollary to Theorem 3.7 we have that $X$ is countably paracompact and normal $[7$, p. 222].

If we take $X$ to be locally compact and $V=C_{0}{ }^{+}(X)$, then $V$ is a Nachbin family on $X$ which satisfies $(\alpha)$ and $C V_{0}(X)=\left(C_{b}(X), \beta\right)$ (see [19]). Moreover, if $F$ is a closed subspace of $X$, then clearly $R_{F}(V) \leqq C_{0}+(F)$. It is also true that $C_{0}{ }^{+}(F) \leqq R_{F}(V)$ as we show below.

3.8. Lemma. The following are equivalent (here it suffices to assume only that $X$ is Hausdorff):

(1) $X$ is locally compact;

(2) For each closed set $F$ in $X$ and for every $\phi \in C_{c}{ }^{+}(F)$ there is a $\theta \in C_{c}+(X)$ such that $R(\phi ; \operatorname{spt}(\phi))=R(\theta ; \operatorname{spt}(\phi))$;

(3) For each closed set $F$ in $X$ and for every $\phi \in C_{0}{ }^{+}(F)$ there is a $\theta \in C_{0}{ }^{+}(X)$ such that $\phi \leqq R(\theta ; F)$.

Proof. Assume that (1) holds, let $F$ be a closed subspace of $X$, and let $\phi \in C_{c}{ }^{+}(F)$. By Stone's theorem $\left[10\right.$, p. 90] there exists $\theta \in C_{b}(X)$ so that $R(\theta ; \operatorname{spt}(\phi))=R(\phi ; \operatorname{spt}(\phi))$, and since $\phi \geqq 0$ we may assume that $\theta \geqq 0$. Moreover, it easily follows from Urysohn's lemma that we can assume that $\theta \in C_{c}+(X)$ so that (2) holds.

Assume that (2) holds, let $F$ be a closed subspace of $X$, let $\phi \in C_{0}+(F)$, and assume that $\|\phi\|_{F}=1$. For $n=1,2, \ldots$, define

$$
K_{n}=\left\{x \in F: 2^{-n} \leqq \phi(x) \leqq 2^{-(n-1)}\right\}
$$

and choose $\phi_{n}=0$ for each $n$ for which $K_{n}$ is void. For those $n$ for which $K_{n}$ is not void, $R\left(\chi_{K_{n}} ; K_{n}\right) \in C_{c}{ }^{+}\left(K_{n}\right)$, and hence there is a $\phi_{n} \in C_{c}{ }^{+}(X)$ such that $R\left(\chi_{K_{n}} ; K_{n}\right)=R\left(\phi_{n} ; K_{n}\right)$ with $\left\|\phi_{n}\right\| \leqq 1$. Consequently,

$$
\theta=\sum_{n=1}^{\infty} 2^{-(n-1)} \phi_{n}
$$

is in $C_{0}+(X)$, while $x \in N(\phi)$ implies that there is an $n$ such that $x \in K_{n}$ so that $\phi(x) \leqq 2^{-(n-1)} \leqq \theta(x)$ and (3) holds.

Now assume that (3) holds and choose $y \in X$. Since $F=\{y\}$ is a closed subspace of $X$ and $R\left(\chi_{F} ; F\right) \in C_{0}+(F)$, there exists $\phi \in C_{0}+(X)$ such that $\phi(y) \geqq 1$ which implies that $\left\{x \in X: \phi(x) \geqq \frac{1}{2}\right\}$ is the desired compact neighbourhood of $y$. 
3.9. Theorem. If $X$ is locally compact and if $\left(C_{b}(X), \beta\right)$ is fully complete, then $X$ is normal and $\left(C_{b}(F), \beta\right)$ is fully complete for every closed subspace $F$ of $X$.

Proof. As we have already noted, $\left(C_{b}(X), \beta\right)=C V_{0}(X)$ where $V=C_{0}{ }^{+}(X)$; therefore, $X$ is normal by Theorem 3.5. Moreover, if $F$ is a closed subspace of $X$, then $C R_{F}(V)_{0}(F)$ is fully complete by Theorem 3.6. In view of $[19$, Corollary 3.2], it will suffice to show that $R_{F}(V) \approx C_{0}+(F)$, and this is immediate from Lemma 3.8.

If $X$ is compact, then $\left(C_{b}(X), \beta\right)$ is a Banach space and hence is fully complete by the Krein-Smulian theorem [8, p. 539]. If, however, $X$ is locally compact but not compact, then $\beta$ is never metrizable $[\mathbf{1}$, p. 98] and the only known example in this case where $\left(C_{b}(X), \beta\right)$ is fully complete is when $X$ is discrete $[4, \mathrm{p} .367]$. It would be of considerable interest to discover necessary and sufficient conditions on $X$ which would imply that $\left(C_{b}(X), \beta\right)$ (or $(C(X)$, co-op)) is fully complete. Theorem 3.5 is a step in this direction, as is the following immediate consequence of Theorem 3.7 (Theorem 3.9).

3.10. Corollary. If a topological property for $X$ is necessary in order that ( $C(X)$, co-op), $\left(\left(C_{b}(X), \beta\right)\right.$ where $X$ is locally compact $)$ be fully complete, then this property is hereditary with respect to closed subspaces of $X$.

It would be of interest just to determine a class of locally compact Hausdorff spaces other than the compact or discrete spaces for which $\left(C_{b}(X), \beta\right)$ is fully complete. In view of the result for discrete spaces, one might reasonably conjecture that the class of locally compact Hausdorff and extremally disconnected spaces would be such a class. Assuming the continuum hypothesis, however, this is not the case. In fact, if $X=\beta N \backslash\{p\}$ where $p \in \beta N \backslash N$ and $X$ has the relative topology from $\beta N$, then $X$ is a locally compact Hausdorff space which is both extremally disconnected and pseudo-compact. But $S=(\beta N \backslash N) \cap X$ is a closed subspace of $X$ which is not $C^{*}$-embedded in $X$ [9, p. 379], and thus $X$ is not normal (for further details, see [21]).

4. $B_{r}$-completeness in certain function spaces. In this section we will obtain several conditions which are necessary and sufficient for $\left(C_{b}(X), \beta\right)$ to be $B_{r}$-complete when $X$ is locally compact and $\sigma$-compact. Before introducing these results, some remarks are in order concerning the relationship between our Nachbin families and the sets of weights considered by Nachbin [14].

First, Nachbin families are structured so as to permit utilization of the partial order $\leqq$ defined in $\S 2$; that this has been a useful device is amply illustrated in this paper, as well as in $[\mathbf{1 9} ; \mathbf{2 0}]$. It is easy to check, however, that the class of weighted spaces obtained by Nachbin's approach is precisely that which is obtained by our approach via Nachbin families. In extending Nachbin's concept of a set of weights to our Nachbin families, however, we have in a sense increased the number of seminorms used in generating the 
weighted topology, and a possible drawback to this is in recognizing when $\omega$ is metrizable. This difficulty is remedied by the following theorem.

4.1. Theorem. Let $V$ be a Nachbin family on $X$. If $U$ is a countable set of non-negative u.s.c. functions on $X$ such that $W=\{\lambda u: \lambda \geqq 0, u \in U\}$ is a Nachbin family on $X$ with $W \approx V$, then $C V_{0}(X)$ is metrizable.

Proof. It suffices to show that $C W_{0}(X)$ is metrizable in view of $[\mathbf{1 9}$, Corollary $3.2]$, and this is immediate from the fact that $U$ is countable.

4.2. Corollary. If in the preceding theorem $U$ is finite, then $C V_{0}(X)$ is normable.

We now proceed to the main result of this section. Recall that Buck [1] proved that the dual of $\left(C_{b}(X), \beta\right)$ was the space $M_{b}(X)$ of bounded Radon measures on $X$ where $X$ is locally compact. Also, if $V$ is a Nachbin family on $X$ and $L$ is a linear subspace of $M_{b}(X)$, then $V$ acts on $L$ in a natural way (see $[19 ; 20]$ ) and $L$ will be called a module over $V$ if $V \cdot L \subseteq L$.

4.3. Theorem. If $X$ is locally compact and $\sigma$-compact, then the following are equivalent:

(1) $\left(C_{b}(X), \beta\right)$ is $B_{r}$-complete;

(2) If $V=\left\{\phi \in C_{0}+(X): N(\phi)=X\right\}$, then each $\sigma\left(M_{b}(X), C_{b}(X)\right)$-dense nearly closed linear subspace of $M_{b}(X)$ is a module over $V$;

(3) Each $\sigma\left(M_{b}(X), C_{b}(X)\right)$-dense nearly closed linear subspace of $M_{b}(X)$ is a module over $C_{0}{ }^{+}(X)$;

(4) Each $\sigma\left(M_{b}(X), C_{b}(X)\right)$-dense nearly closed linear subspace of $M_{b}(X)$ is variation norm dense in $M_{b}(X)$.

Proof. We first show that (1) and (4) are equivalent. To do this, let $L$ be a $\sigma\left(M_{b}(X), C_{b}(X)\right)$-dense nearly closed linear subspace of $M_{b}(X)$, and assume that $L$ is variation norm dense in $M_{b}(X)$. Take $\mu \in M_{b}(X)$ and choose $\left\{\mu_{n}\right\}_{n=1}^{\infty}$ in $L$ so that $\mu_{n} \rightarrow \mu$ in variation norm. Therefore $\mu_{n} \rightarrow \mu$ in the $\sigma\left(M_{b}(X), C_{b}(X)\right)$-topology, and this implies that there exists $\phi \in C_{0}+(X)$ such that $\left\{\mu_{n}\right\}_{n=1}^{\infty} \subseteq V_{\phi}^{0}\left[5\right.$, p. 161]. Since $L \cap V_{\phi}^{0}$ is $\sigma\left(M_{b}(X), C_{b}(X)\right)$-closed, $\mu \in L$ and we have that (4) implies (1). Because (1) obviously implies (4), the claim is verified.

It is clear that (3) implies (2), while the fact that (1) implies (3) is immediate from [20, Theorem 5.2]. Therefore the proof will be complete when we show that (2) implies (1). From the fact that $V$ (in (2)) is non-void $\left[1\right.$, p. 96], it readily follows that $V$ is a Nachbin family on $X$ with $V \approx C_{0}+(X)$, and so it will suffice to show that $C V_{0}(X)$ is $B_{r}$-complete whenever (2) holds.

Let $L$ be a $\sigma\left(M_{b}(X), C_{b}(X)\right)$-dense nearly closed linear subspace of $M_{b}(X)$ and assume that $L$ is a module over $V$. If we fix $v \in V$, then $A=\{\lambda v: \lambda \geqq 0\}$ is a Nachbin family on $X$ for which $C A_{0}(X)$ is Hausdorff. Further, $C A_{0}(X)$ is normable from Corollary 4.2 and complete [19, Corollary 3.7], and thus has the Krein-Smulian property [8, p. 539]. Now [19, Theorem 3.9] states 
that $C A_{0}(X)^{*}=A \cdot M_{b}(X) \subseteq V \cdot M_{b}(X)=C V_{0}(X)^{*}=M_{b}(X)$, while [19, Theorem 3.1] implies that $C V_{0}(X) \subseteq C A_{0}(X)$. We next show that the linear subspace $L_{v}=L \cap A \cdot M_{b}(X)$ of $C A_{0}(X)^{*}$ is $\sigma\left(C A_{0}(X)^{*}, C A_{0}(X)\right)$ dense in $C A_{0}(X)^{*}$, and for this purpose we fix $\mu \in A \cdot M_{b}(X), f \in C A_{0}(X)$, and $\epsilon>0$. Now there exist $\lambda>0, \nu \in M_{b}(X)$ so that $\mu=(\lambda v) \cdot \nu$, and since $f v \in C V_{0}(X)$, we can find $\sigma \in L$ for which $\left|\int f v d \nu-\int f v d \sigma\right|<\lambda^{-1} \epsilon$. Consequently, $(\lambda v) \cdot \sigma \in L_{v}$ and $\left|\int f d \mu-\int f d((\lambda v) \cdot \sigma)\right|<\epsilon$. It is also true that $L_{v}$ is nearly closed in $C A_{0}(X)^{*}$ as we now show. Fix $\lambda>0$, let $u=\lambda v$, and observe that it suffices to show that

$$
B=\left\{f \in C A_{0}(X):\|f u\| \leqq 1\right\}^{0} \cap L_{v}
$$

is $\sigma\left(C A_{0}(X)^{*}, C A_{0}(X)\right)$-closed. Since the injection map $i: C V_{0}(X) \rightarrow C A_{0}(X)$ is continuous, its transpose $i^{*}: A \cdot M_{b}(X) \rightarrow V \cdot M_{b}(X)$ is weak-* continuous, and so

$$
C=\left[\left\{f \in C V_{0}(X):\|f u\| \leqq 1\right\}^{0} \cap L\right] \cap A \cdot M_{b}(X)
$$

is $\sigma\left(C A_{0}(X)^{*}, C A_{0}(X)\right)$-closed. Moreover, it is clear that $B \subseteq C$, while if $\mu \in C$, then $\mu \in L_{v}$ and there is a $\nu \in M_{b}(X)$ with $\|\nu\| \leqq 1$ such that $\mu=u \cdot \nu\left[20\right.$, Theorem 4.5]. Therefore $B=C$, since if $f \in C A_{0}(X)$ with $\|f u\| \leqq 1$, then $\left|\int f d \mu\right|=\left|\int f u d \nu\right| \leqq\|f u\| \cdot\|\nu\| \leqq 1$ which implies that $\mu \in B$. It now follows that $L_{v}=A \cdot M_{b}(X)$, and hence

$$
M_{b}(X)=V \cdot M_{b}(X) \subseteq \cup\left\{L_{v}: v \in V\right\} \subseteq L
$$

which yields that $L=M_{b}(X)$ and completes the proof.

We conclude this section by remarking that it is not yet known whether or not $\left(C_{b}(\mathbf{R}), \beta\right)$ is $B_{r}$-complete, much less fully complete.

5. Products and tensor products. The permanence properties of fully complete spaces were considered first by Collins, and in his paper [2] which appeared in 1955 it is shown that an arbitrary product of fully complete spaces need not be fully complete. In 1958, Kelley [13] introduced the notion of hypercomplete spaces (a priori, fully complete), and conjectured that countable products of hypercomplete spaces would be hypercomplete. We resolve this conjecture (see [18]), as well as consider other aspects of completeness properties in products and tensor products of locally convex spaces.

5.1. Theorem. If $E$ is a Fréchet space and if $F=\left(E^{*}, \tau\left(E^{*}, E\right)\right)$, then the direct sum of countably many copies of $F$ has the Krein-Smulian property, and hence is both hypercomplete and fully complete.

Proof. For $A \subseteq N$ (natural numbers), let $E_{\alpha}=E, F_{\alpha}=F$ for each $\alpha \in A$, let $G=\prod_{\alpha \in A} E_{\alpha}$, and note that it suffices to show that $\sum_{\alpha \in A} F_{\alpha}$ has the Krein-Smulian property. Now $\sum_{\alpha \in A} F_{\alpha}=G^{*}$ and $\tau\left(G^{*}, G\right)$ is the direct sum topology on $G^{*}\left[\mathbf{1 7}\right.$, p. 137], so that $\sum_{\alpha \in A} F_{\alpha}$ is the dual of a Fréchet space and has the Mackey topology. But such a space has the Krein-Smulian 
property, as can be shown by a simple modification of an argument in $[8$, p. 540].

From $\left[6\right.$, p. 478], the strict topology $\beta$ on $l^{\infty}$ is $\tau\left(l^{\infty}, l^{1}\right)$. This gives us the following corollary to Theorem 5.1 which includes as a special case the result due to Collins $\left[4\right.$, p. 367] that $\left(l^{\infty}, \beta\right)$ has the Krein-Smulian property.

5.2. Corollary. The direct sum of countably many copies of $\left(l^{\infty}, \beta\right)$ has the Krein-Smulian property.

It is well known that, in general, countable direct sums of spaces with the Krein-Smulian property fail to be fully complete (see, e.g., [12, p. 69]). This follows, in fact, from an example due to Grothendieck [11, p. 92], and it is this example which suggested the following result.

5.3. Theorem. If $E$ is the direct sum of denumerably many copies of $\left(l^{\infty}, \beta\right)$ and if $F$ is the product of denumerably many copies of $c_{0}$, each endowed with the uniform topology, then both $E$ and $F$ have the Krein-Smulian property, but $E \times F$ is not fully complete.

Proof. $E$ has the Krein-Smulian property from Corollary 5.2, while the Krein-Smulian theorem $[\mathbf{8}$, p. 539] guarantees this property for $F$. To see that $E \times F$ is not fully complete, define $G$ to be the product of denumerably many copies of $\left(l^{\infty}, \beta\right)$ and then define $t: E \times F \rightarrow G$ by $t(x, y)=x+y$. One routinely verifies that $t$ is a linear mapping whose range is a proper (dense) subspace of $G$ and that $t$ is continuous and nearly open. In view of Theorem 2.1, we have that $E \times F$ is not fully complete.

It should be noted that since a Fréchet space has the Krein-Smulian property, $E \times F$ in Theorem 5.3 can be considered as a product of spaces with the Krein-Smulian property of any finite or denumerable order. Theorem 5.3 , of course, resolves Kelley's conjecture, but the question of whether or not $B_{r}$-completeness is preserved by products is still open.

It is an immediate consequence of Theorem 2.2 that if the product of two locally convex spaces $E$ and $F$ is fully complete, then both $E$ and $F$ are also fully complete. Surprisingly enough, an analogous result also holds for certain tensor products as we show below.

5.4. Theorem. Let $X$ and $Y$ be locally compact, let $U$ be a Nachbin family on $X$ with $C_{c}+(X) \leqq U \leqq B^{+}(X)$, and let $V$ be a Nachbin family on $Y$ with $C_{c}+(Y) \leqq V \leqq B^{+}(Y)$. If the biequicontinuous completed tensor product $C U_{0}(X) \otimes \approx C V_{0}(Y)$ is fully complete, then both $C U_{0}(X)$ and $C V_{0}(Y)$ are fully complete.

Proof. In view of our representation theorem [20, Theorem 5.1], it follows from Theorem 2.2 that $C W_{0}(X \times Y)$ is fully complete where

$$
W=U \times V=\{u \times v: u \in U, v \in V\}
$$


(here $u \times v$ is the function on $X \times Y$ defined by $u \times v(x, y)=u(x) v(y)$ ). Since $W$ is a Nachbin family on $X \times Y$ which satisfies $(\alpha)$ and since $W \leqq B^{+}(X \times Y)$, we have from Theorem 3.6 that $C R_{F}(W)_{0}(F)$ is fully complete for any closed subspace $F$ of $X \times Y$. Fix $z \in Y$, let $F$ denote the closed subspace $X \times\{z\}$, and define $t: C R_{F}(W)_{0}(F) \rightarrow C(X)$ by $t(f)(x)=$ $f(x, z)$. Clearly, $t$ is a well-defined linear map, and since there is a $v \in V$ such that $v(z)>0$, it follows that $t$ maps into $C U_{0}(X)[14$, p. 68]. We will show that $t$ is onto, and to this end we take $f \in C U_{0}(X)$, define $g \in C(F)$ by $g(x, z)=f(x)$, and show that $g \in C R_{F}(W)_{0}(F)$. To do this, let $u \in U, v \in V$, let $\epsilon>0$, and choose $w \in U$ so that $v(z) u \leqq w$. It suffices to show that $G=\{(x, z):|g(x, z)| u(x) v(z) \geqq \epsilon\}$ is compact, and this is the case since

$$
G \subseteq\{x \in X:|f(x)| w(x) \geqq \epsilon\} \times\{z\} .
$$

If $\left\{f_{i}\right\}$ is a net in $C R_{F}(W)_{0}(F)$ such that $f_{i} \rightarrow o(\omega)$, then for $u \in U$ and $v \in V$ with $v(z) \geqq 1$ we have

$$
\left|t\left(f_{i}\right)(x)\right| u(x) \leqq\left|f_{i}(x, z)\right| u(x) v(z) \leqq\left\|f_{i}(u \times v)\right\|_{F} .
$$

Consequently, $t$ is a continuous linear map of $C R_{F}(W)_{0}(F)$ on to $C U_{0}(X)$ which is clearly one-to-one. Moreover, if $\left\{f_{i}\right\}$ is a net in $C U_{0}(X)$ such that $f_{i} \rightarrow o\left(\omega_{U}\right)$, then for $u \in U, v \in V$, and $w \in U$ with $v(z) u \leqq w$ we have

$$
\left|t^{-1}\left(f_{i}\right)(x, z)\right| u(x) v(z) \leqq\left|f_{i}(x)\right| w(x) \leqq \| f_{i} w||,
$$

and this implies that $t^{-1}$ is continuous. Theorem 2.2 now tells us that $C U_{0}(X)$ is fully complete; similarly, $C V_{0}(Y)$ is fully complete, and this concludes the proof.

It is not known whether the converse of the preceding theorem is true. However, it is our conjecture that it, like the analogous question concerning products of fully complete spaces discussed in the beginning of this section, is false. We conclude with a remark which could prove useful in resolving this conjecture.

Let us denote the class of all locally convex spaces which are fully complete by $\mathscr{C}$, and assume it true that whenever $E, F \in \mathscr{C}$, then $E \otimes \approx F \in \mathscr{C}$ (for the conclusion, we may assume that $F$ is a Banach space). This hypothesis then gives us the following result:

If $X$ is locally compact, if $V$ is a Nachbin family on $X$ with

$$
C_{c}{ }^{+}(X) \leqq V \leqq C_{0}{ }^{+}(X),
$$

and if $C V_{0}(X) \in \mathscr{C}$, then $X$ is paracompact.

This is so since in this case $C W_{0}(X \times \beta X) \in \mathscr{C}$ from [20, Theorem 5.1] and Theorem 2.2 where $W=V \times C^{+}(\beta X)$; it follows from [19, Lemma 4.6] that $W \leqq C_{0}{ }^{+}(X \times \beta X)$, and this implies that $X \times \beta X$ is normal by Theorem 3.5 whence $X$ is paracompact [22, p. 1046]. 


\section{REFERENCES}

1. R. C. Buck, Bounded continuous functions on a locally compact space, Michigan Math. J. o (1958), 95-104.

2. H. S. Collins, Completeness and compactness in linear topological spaces, Trans. Amer. Math. Soc. 79 (1955), 256-280.

3. - Completeness, full completeness, and $k$ spaces, Proc. Amer. Math. Soc. 6 (1955), 832-835.

4. - On the space $l^{\infty}(S)$, with the strict topology, Math. Z. 106 (1968), 361-373.

5. H. S. Collins and J. R. Dorroh, Remarks on certain function spaces, Math. Ann. 176 (1968), $157-168$.

6. J. B. Conway, The strict topology and compactness in the space of measures, Trans. Amer. Math. Soc. 126 (1967), 474-486.

7. C. H. Dowker, On countably paracompact spaces, Can. J. Math. 3 (1951), 219-224.

8. R. E. Edwards, Functional analysis (Holt, Rinehart and Winston, New York, 1965).

9. N. J. Fine and L. Gillman, Extensions of continuous functions in $\beta N$, Bull. Amer. Math. Soc. 66 (1960), 376-381.

10. L. Gillman and M. Jerison, Rings of continuous functions (Van Nostrand, Princeton, N. J., 1960).

11. A. Grothendieck, Sur les espaces $(F)$ et $(D F)$, Summa Brasil Math. 3 (1954), 57-123.

12. T. Husain, The open mapping and closed graph theorems in topological rector spaces (Oxford Univ. Press, Oxford, 1965).

13. J. L. Kelley, Hypercomplete linear topological spaces, Michigan Math. J. 5 (1958), 235-246.

14. L. Nachbin, Elements of approximation theory (Van Nostrand, Princeton, N. J., 1967).

15. V. Pták, On complete topological linear spaces, Czechoslovak Math. J. 78 (1953), 301-364.

16. - Completeness and the open mapping theorem, Bull. Soc. Math. France 86 (1958), 41-74.

17. H. H. Schaefer, Topological vector spaces (MacMillan, New York, 1966).

18. W. H. Summers, Products of fully complete spaces, Bull. Amer. Math. Soc. 75 (1969), 1005.

19. - A representation theorem for biequicontinuous completed tensor products of weighted spaces, Trans. Amer. Math. Soc. 146 (1969), 121-131.

20. - Dual spaces of weighted spaces (to appear in Trans. Amer. Math. Soc.).

21. - On the full-completeness of certain function spaces (to appear).

22. H. Tamano, On paracompactness, Pacific J. Math. 10 (1960), 1043-1047.

23. S. Warner, The topology of compact convergence on continuous function spaces, Duke Math. J. 25 (1958), 265-282.

University of Arkansas,

Fayetterille, Arkansas 